\title{
Acquisition of 5-fluorouracil resistance induces epithelial-mesenchymal transitions through the Hedgehog signaling pathway in HCT-8 colon cancer cells
}

\author{
YANJUN LIU ${ }^{1 *}$, FANGFANG DU ${ }^{2 *}$, QIANNAN ZHAO ${ }^{2}$, JIAN JIN $^{2}$, XIN MA $^{2}$ and HUAZHONG LI ${ }^{1}$ \\ ${ }^{1}$ School of Biotechnology, ${ }^{2}$ School of Pharmaceutical Sciences, Jiangnan University, Wuxi, Jiangsu 214122, P.R. China
}

Received May 26, 2014; Accepted February 2, 2015

DOI: $10.3892 / \mathrm{ol} .2015 .3136$

\begin{abstract}
Colon cancer has a high incidence in individuals $>60$-years-old. The commonly used chemotherapeutic agent, 5-fluorouracil (5-FU), has gradually lost its potency in treating colorectal cancer following the acquisition of resistance. Drug resistance is usually associated with epithelial-mesenchymal transitions (EMTs) in cancer cells. In the present study, the EMT phenotypes of two colon cancer cell lines, wild-type (HCT-8/WT) and 5-FU-resistant (HCT-8/5-FU), were characterized following the analysis of cellular migration, proliferation, morphology and molecular changes. In order to further clarify the mechanism of EMT in HCT-8/5-FU cells, the effect of EMT pathway inhibitors upon drug sensitivity was investigated. The results revealed that the Hedgehog signaling pathway inhibitor, GDC0449, reversed drug resistance. Therefore, inhibition of the Hedgehog pathway may provide a novel chemotherapeutic strategy for the treatment of patients with 5-FU-resistant colon cancer.
\end{abstract}

\section{Introduction}

As a result of early diagnosis and improved treatment strategies, the survival rates of colon cancer patients have increased (1); however, the five-year survival rate remains at $<60 \%$ (2). For colon cancer in situ, surgery is the primary curative method. However, in the later phases (e.g. node-positive stage III), adjuvant chemotherapy is required (1). As a first-line chemotherapeutic agent for colon cancer,

Correspondence to: Professor Xin Ma, School of Pharmaceutical Sciences, Jiangnan University, 1800 Lihu Road, Wuxi, Jiangsu 214122, P.R. China

E-mail: maxin@jiangnan.edu.cn

Professor Huazhong Li, School of Biotechnology, Jiangnan University, 1800 Lihu Road, Wuxi, Jiangu 214122, P.R. China

E-mail: hzhli@jiangnan.edu.cn

${ }^{*}$ Contributed equally

Key words: colon cancer, epithelial-mesenchymal transition, Hedgehog pathway 5-fluorouracil (5-FU) is administered in order to increase the likelihood of survival (3). The structure of 5-FU resembles the pyrimidines of DNA and RNA (4); therefore, it is able to disrupt nucleoside metabolism and be incorporated into RNA and DNA molecules. This results in cell-cycle arrest at $G_{1}$ phase and at the $G_{1} / S$ boundary, which prolongs DNA synthesis (5-7). Despite its anticancer effects, the clinical use of 5-FU is limited by drug resistance. 5-FU used alone, or in combination with other antitumor drugs, has a relatively low response rate in the treatment of colon cancer $(8,9)$. Therefore, the mechanisms underlying 5-FU resistance require further investigation.

Epithelial-mesenchymal transition (EMT) is a process in which epithelial cells lose a number of endogenous characteristics and acquire typical features of mesenchymal cells (10). For instance, the cobblestone appearance of epithelial cells changes to a spindle-like shape. In addition, epithelial biomarkers (including E-cadherin) are lost, while mesenchymal markers (such as N-cadherin, vimentin and Snail) are acquired (11). EMT in cancer cells has been identified to facilitate metastasis and chemoresistance (12). Furthermore, an EMT phenomenon has been identified in a number of cancer cells, including cisplatin-resistant non-small-cell lung cancer (13), doxorubicin-resistant breast cancer (14) and sorafenib-resistant hepatocellular carcinoma cells (15). Preventing EMT-associated signaling pathways plays an important role in inhibiting cancer cell migration and invasion, as well as reducing drug resistance (12). The occurrence of the EMT is associated with complex signaling pathways, including the Wnt, PI3K, Hedgehog, transforming growth factor- $\beta$ (TGF- $\beta$ ) and Notch pathways (16-18). However, the details of these mechanisms in HCT-8 colon cancer cells are yet to be elucidated.

In order to identify potential therapeutic targets for the treatment of 5-FU-resistant colon cancer, the present study investigated the pathway(s) involved in the induction of an EMT phenotype and the acquisition of 5-FU resistance in HCT-8/5-FU cells.

\section{Materials and methods}

Cells and reagents. The HCT-8/wild-type (WT) and HCT-8/5-FU cell lines were obtained from KeyGen Biotech Co. Ltd. (Nanjing, China) and cultured in RPMI-1640 containing $10 \%$ fetal bovine 
serum (FBS), $100 \mathrm{U} / \mathrm{ml}$ penicillin (Beyotime Institute of Biotechnology, Shanghai, China) and $100 \mathrm{U} / \mathrm{ml}$ streptomycin (Beyotime Institute of Biotechnology) at $37^{\circ} \mathrm{C}$ under an atmosphere of $5 \% \mathrm{CO}_{2}$. In order to maintain drug resistance, $15 \mu \mathrm{g} / \mathrm{ml} 5-\mathrm{FU}$ was added to the HCT-8/5-FU cells. The 5-FU and GDC0449 agents were purchased from King York Co. Ltd. (Tianjin, China) and Selleck Chemicals (Houston, TX, USA), respectively.

Immunofluorescent staining. The cells were fixed in $4 \%$ paraformaldehyde for $30 \mathrm{~min}$, permeated with $0.1 \%$ Triton X-100 [Sangon Biotech (Shanghai) Co., Ltd., Shanghai, China] for $10 \mathrm{~min}$ at room temperature and then washed three times with $0.05 \%$ Tween-20. After blocking with $5 \%$ bovine serum albumin (BSA) in phosphate-buffered saline (PBS) for $30 \mathrm{~min}$, primary monoclonal rabbit anti-human E-cadherin (dilution, 1:200; cat. no. 1702-1; Epitomics, Inc., Burlingame, CA, USA) and monoclonal mouse anti-rabbit vimentin (dilution, 1:500; cat. no. ab8069; Abcam, Cambridge, UK) antibodies were added and incubated at $4^{\circ} \mathrm{C}$ overnight. Next, fluorescent donkey anti-rabbit Alexa Fluor 568-conjugated (dilution, 1:200; Invitrogen Life Technologies, Carlsbad, CA, USA) or donkey anti-mouse Alexa Fluor 488-conjugated (dilution, 1:200; Invitrogen Life Technologies) secondary antibodies were added for $1 \mathrm{~h}$ at room temperature. DAPI (Beyotime Institute of Biotechnology) was used to stain the nuclei of the cells. Images were captured using a confocal laser scanning microscope (TCS SP8; Leica Microsystems GmBH, Wetzlar, Germany).

Flow cytometry. The cells were collected by centrifugation at $500 \mathrm{x}$ g for $5 \mathrm{~min}$, blocked with 5\% BSA in PBS for 30 min (for vimentin, this process was the same as for the immunofluorescent staining) and incubated with the antibodies for $30 \mathrm{~min}$ at $4^{\circ} \mathrm{C}$. Subsequent to each treatment, the cells were washed with $2 \%$ BSA in PBS and collected by centrifugation at $500 \mathrm{x}$ g for $5 \mathrm{~min}$. Fluorescence was detected using a FACSCalibur flow cytometer (BD Biosciences, Franklin, NJ, USA).

Wound-healing assay. Cellular motility was assessed using a wound repair assay, as described previously (19-21). Briefly, the cells were plated at a density of $1 \times 10^{4}$ cells per well in 24-well plates and incubated overnight in serum-free medium. A straight line was then scratched through the attached cells using a sterile tip. The suspended cells were removed, and medium containing $2 \%$ FBS was added. Images were then captured immediately $(0 \mathrm{~h})$ and at $48 \mathrm{~h}$ using a fluorescence microscope (TS100, Nikon Corporation, Tokyo, Japan). The width of the wound was measured using ImageJ software (National Institutes of Health, Bethesda, MD, USA) in order to determine the extent of migration.

Migration assay. Transwell chamber inserts were used in order to investigate cellular migration in vitro. A $100 \mu \mathrm{l}$ serum-free suspension containing $5 \times 10^{4}$ cells was added to a chamber with $8-\mu \mathrm{m}$ pores (BD Biosciences). Next, the chamber was placed in a 24 -well plate containing $10 \%$ FBS. Subsequent to a 24-h incubation, a number of cells had migrated to the lower surface. These cells were stained with crystal violet [Sangon Biotech (Shanghai) Co., Ltd.] and images were captured using a Leica CME microscope (Leica Microsystems $\mathrm{GmBH}$ ) and a
Nikon Coolpix 54 camera (Nikon Corporation). The migrated cells were counted in randomly-selected fields.

5-FU chemosensitivity assay. The cells were plated into 96 -well plates at a density of $8 \times 10^{3}$ cells per well. Following attachment, a series of 5-FU concentrations $(2.5-20,000 \mu \mathrm{g} / \mathrm{ml}$ in two-fold serial dilutions) were added. The plates were then incubated for $48 \mathrm{~h}$ at $37^{\circ} \mathrm{C}$ under $5 \% \mathrm{CO}_{2}$. Next, $10 \mu \mathrm{l}$ MTT ( $5 \mathrm{mg} / \mathrm{ml}$; Sigma-Aldrich, St. Louis, MO, USA) was added, and the plates were incubated for a further $4 \mathrm{~h}$. Subsequently, $150 \mu 1$ dimethyl sulfoxide was added in order to dissolve the formazan crystals. Finally, the absorbance was measured at $492 \mathrm{~nm}$ using a microplate reader (Multiskan MK3; Thermo Fisher Scientific, Waltham, MA, USA).

Cell proliferation assay. Cellular proliferation was analyzed at an absorbance of $492 \mathrm{~nm}$. The cells were seeded into 96-well plates at a density of $3 \times 10^{3}, 5 \times 10^{3}$ or $1 \times 10^{4}$ cells per well. Subsequent to a $24 \mathrm{~h}$ incubation, the absorbance was measured using an MTT assay. In addition, cells were plated at a density of $5 \times 10^{3}$ cells per well in 96-well plates, and the absorbance was measured at 24, 48, 72 and $96 \mathrm{~h}$ by MTT assay.

Reverse transcription-polymerase chain reaction (RT-PCR). Total RNA was extracted for the reverse transcription reactions using Reverse Transcriptase M-MLV (Takara Bio Inc., Otsu, Japan) and the Oligo $\mathrm{dT}_{18}$ primer (Takara Bio Inc.). HCT-8/5-FU cells were exposed to $5 \mu \mathrm{M}$ GDC0449 for $48 \mathrm{~h}$. Then the total RNA was extracted. Next, the cDNA was used in the PCR analysis with $2 \mathrm{X}$ Taq Master Mix (25 $\mu \mathrm{l})$, containing $0.2 \mathrm{mM}$ dNTP mixture and 2X PCR buffer (20 mM Tris-HCl, pH 8.3, $100 \mathrm{mM} \mathrm{KCl,} 3 \mathrm{mM} \mathrm{MgCl}{ }_{2}$; Takara Bio Inc.). The PCR conditions were as follows: $95^{\circ} \mathrm{C}$ for $30 \mathrm{sec}, 60^{\circ} \mathrm{C}$ for $30 \mathrm{sec}$ and $72^{\circ} \mathrm{C}$ for $30 \mathrm{sec}$, for 34 cycles followed by a subsequent elongation step at $72^{\circ} \mathrm{C}$ for $10 \mathrm{~min}$. The PCR results were visualized on agarose gel. The images were analyzed using ImageJ software. $\beta$-actin was used as an endogenous control. The primer sequences were as follows: $\beta$-actin forward, 5'-TGAAGTGTGACGTGGACATC-3', and reverse, 5'-GGA GGAGCAATGATCTTGAT-3'; N-cadherin forward, 5'-ACA GTGGCCACCTACAAAGG-3', and reverse, 5'-TGATCCCTC AGGAACTGTCC-3'.

Statistical analysis. The results are presented as the mean \pm standard deviation. Statistical differences were determined using Student's t-test. All statistical analyses were performed using GraphPad Prism version 5.01 software (GraphPad Software, Inc., La Jolla, CA, USA). A value of $\mathrm{P}<0.05$ was considered to indicate a statistically significant difference.

\section{Results}

HCT-8/5-FU cells develop morphological and molecular changes consistent with EMT signaling. The HCT-8/WT cells grew in clusters in vitro, which is a typical feature of the epithelial phenotype. By contrast, the HCT-8/5-FU cells were scattered and had few connections as a result of the presence of $15 \mu \mathrm{g} / \mathrm{ml} \mathrm{5-FU} \mathrm{(Fig.} \mathrm{1A).}$

EMT-associated biomarkers were assessed in order to determine whether the acquisition of 5-FU resistance induced 
A
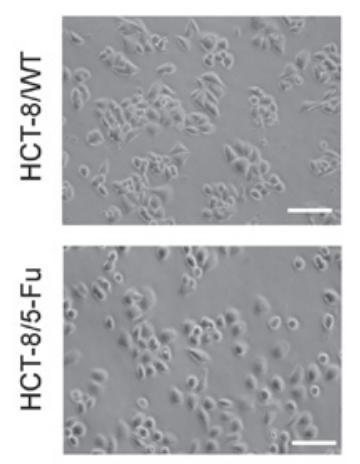

B
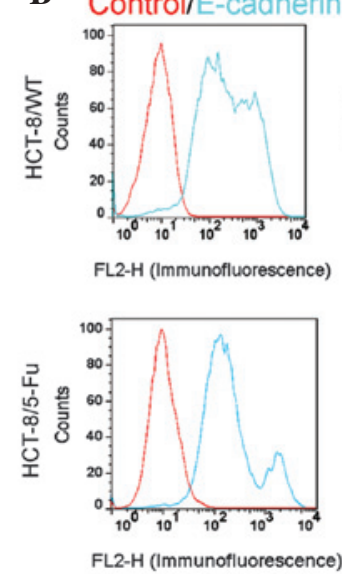

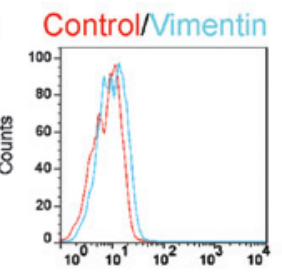

FL2-H (Immunofluorescence)

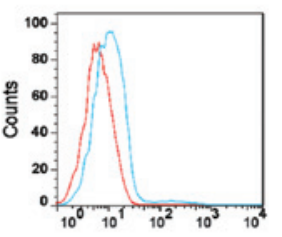

FL2-H (Immunofluorescence)

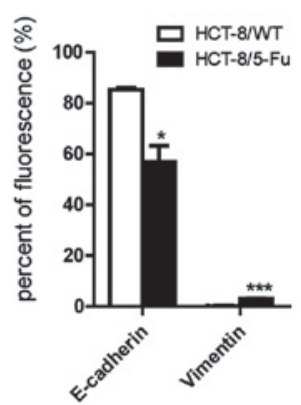

C
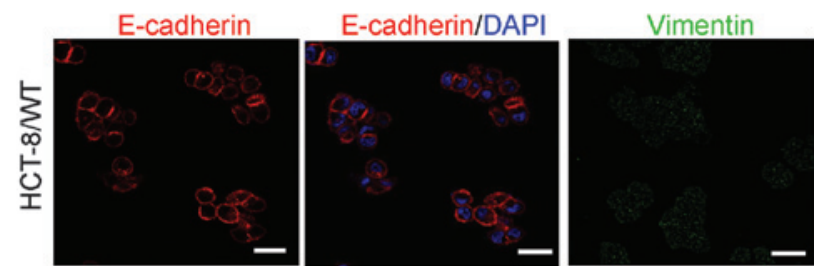

Vimentin/DAPI
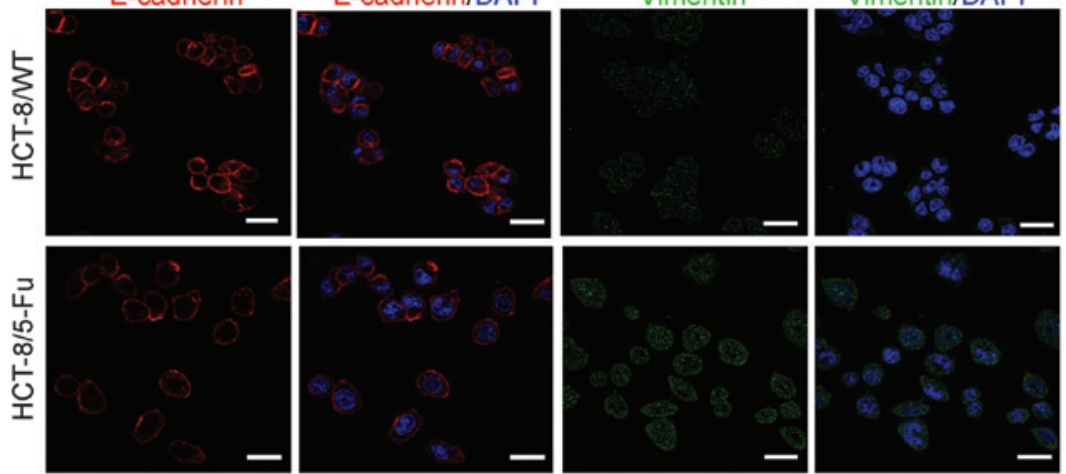

Figure 1. EMT emerges in the 5-FU resistant HCT-8/5-FU cell line morphologically, and at the molecular level. (A) HCT-8/WT cells grew in clusters and exhibited adhesion between cells (upper panel), whereas HCT-8/5-FU cells were scattered and lacked adhesion (lower panel). Scale bars, $100 \mu \mathrm{m}$. (B) Representative cytometric data (left panels) and statistics (right panel) revealing the expression of E-cadherin and vimentin in HCT-8/WT and HCT-8/5-FU cells. ${ }^{*}$ P $<0.05$ compared with the HCT-8/WT cells. (C) Confocal images of the cultured HCT-8 cell lines stained for the EMT-associated proteins, E-cadherin and vimentin. The nuclei were stained with DAPI. Scale bars, $25 \mu \mathrm{m}$. 5-FU, 5-fluorouracil; EMT, epithelial-mesenchymal transition; DAPI, diamidino-2-phenylindole.

$\mathbf{A}$
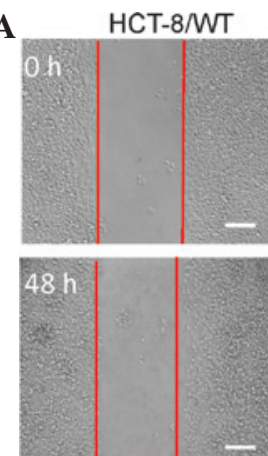

HCT-8/5-Fu

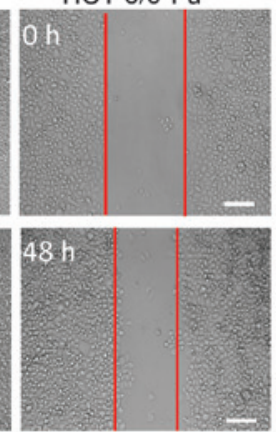

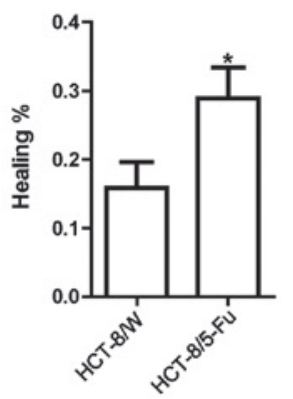

B
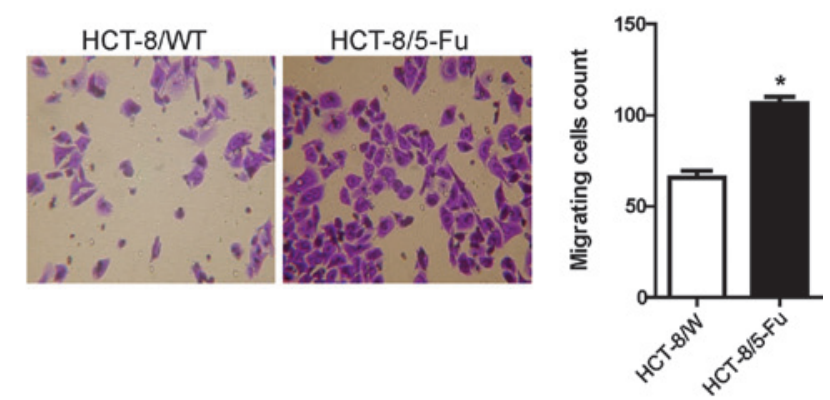

D

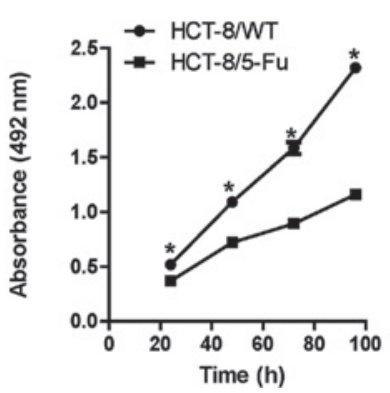

Figure 2. HCT-8/5-FU cells exhibited high migration but low proliferation rates compared with the HCT-8/WT cells. (A) The HCT-8/5-FU cells exhibited a faster migration at $48 \mathrm{~h}$ on the wound scratch assay compared with the HCT-8/WT cells. Red bars indicate the borders of the scratch. Scale bars, $100 \mu \mathrm{m}$. (B) Images from the Transwell chamber insert assays (left panels) and a summary of the migrating cell counts (right panel). "P<0.05 vs. the HCT-8/WT cells. (C) HCT-8/WT cell-proliferation assays with various cell numbers at $48 \mathrm{~h}$ and (D) the growth curve for HCT-8/WT cells at various time points revealed increases compared with HCT- $8 / 5$-FU cells. ${ }^{~} \mathrm{P}<0.05$ vs. the HCT-8/5-FU cells. 
A

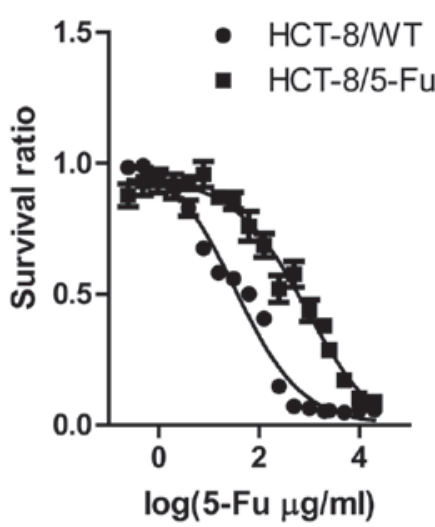

B

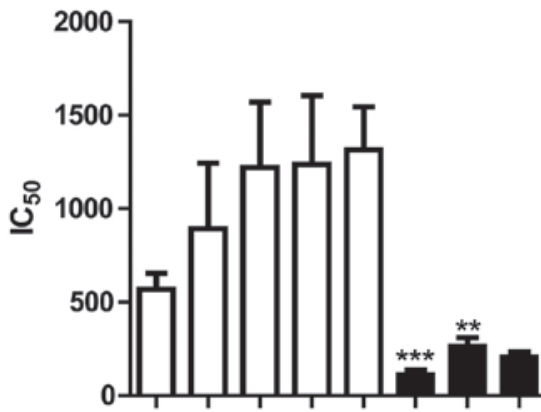

C

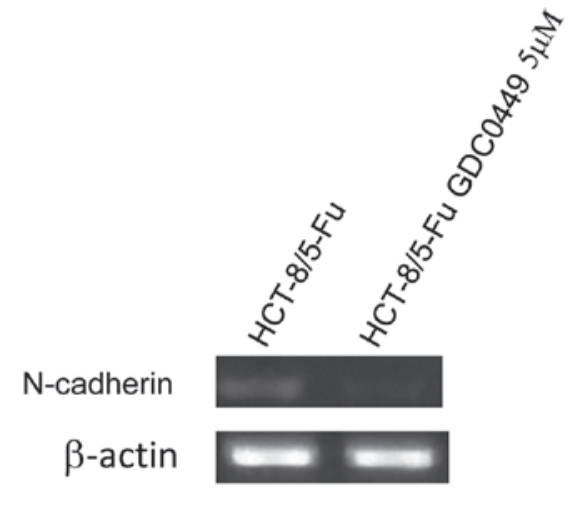

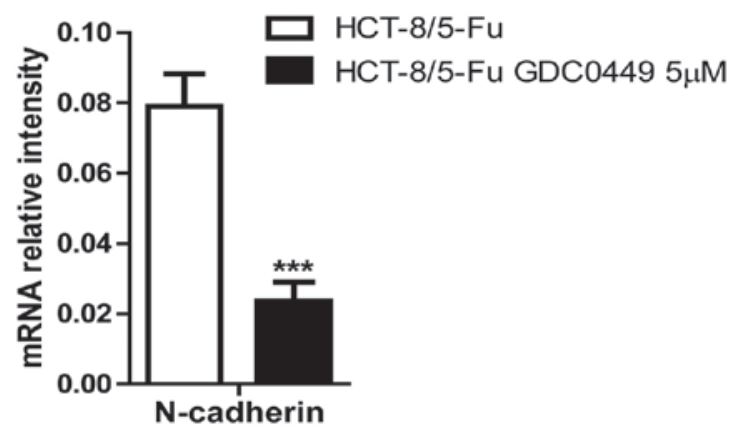

Figure 3. MTT assay revealing that a specific inhibitor of Hedgehog, GDC0449, reversed drug-resistance and EMT in HCT-8/5-FU cells. (A) MTT assays revealing a high resistance to 5-FU of HCT-8/5-FU cells compared with the HCT-8/WT cells. (B) Inhibition of the EMT-associated Hedgehog signaling pathway using GDC0449 led to an increased cellular sensitivity to 5-FU. The optimal concentration of GDC0449 was $5 \mu$ M. (C) RT-PCR results revealing a decrease in the EMT-associated biomarker, N-cadherin, in HCT-8/5-FU cells treated with GDC0449 (left panel). Statistics of RT-PCR (right panel). "P<0.05. EMT, epithelial-mesenchymal transition; 5-FU, 5-fluorouracil; RT-PCR, reverse transcription polymerase chain reaction.

EMT changes on the molecular level. Compared with the HCT-8/WT cells, HCT-8/5-FU cells demonstrated a significant reduction in the level of E-cadherin and an upregulation in the expression of vimentin (Fig. 1B-D).

HCT-8/5-FU cells exhibit increased migration but reduced proliferation. The HCT-8/5-FU cells exhibited a higher capacity for wound-healing compared with the HCT-8/WT cells (Fig. 2A). In addition, the number of migrated HCT-8/5-FU cells was significantly higher compared with the HCT-8/WT cells (Fig. 2B).

The multiplication capacity of the two HCT- 8 cell lines was investigated using a modified MTT assay. Different numbers of HCT-8/WT cells demonstrated increased proliferation following a 48-h plating period compared with the HCT-8/5-FU cells (Fig. 2C). Similar results were observed for the proliferation assay at various times (Fig. 2D).

GDC0449, an inhibitor of the Hedgehog signaling pathway, reverses drug resistance and EMT in HCT-8/FU cells. MTT assays were performed in order to analyze 5-FU resistance in the cell lines. The half maximal inhibitory concentration $\left(\mathrm{IC}_{50}\right)$ of the HCT-8/5-FU cells $(899.2 \mu \mathrm{g} / \mathrm{ml})$ was $\sim 26$-fold higher compared with that of HCT-8/WT cells (33.53 $\mu \mathrm{g} / \mathrm{ml})$ (Fig. 3A).

Next, drug sensitivity was assessed following exposure of HCT-8/5-FU cells to inhibitors of EMT-associated signaling pathways. The Hedgehog pathway inhibitor, GDC0449, was found to reverse drug resistance (Fig. 3B). The optimum concentration of GDC0449 was $5 \mu \mathrm{M}$, which decreased the $\mathrm{IC}_{50}$ by $\sim 8$-fold compared with the untreated HCT-8/5-FU cells (Fig. 3B).

RT-PCR was performed in order to confirm whether the inhibitor, GDC0449, reversed the EMT signals. The EMT-associated biomarker, $\mathrm{N}$-cadherin, was downregulated following treatment with GDC0449 (Fig. 3C).

\section{Discussion}

Chemoresistance limits the effectiveness of colon cancer treatment. Therefore, it is important to identify the mechanisms that are involved in drug resistance. The present study used wild-type (HCT-8/WT) and 5-FU-resistant (HCT-8/5-FU) colorectal cancer cells to investigate the molecular mechanisms and cellular behaviors involved in 5-FU resistance.

The present study verified that HCT-8/5-FU cells undergo EMT processes based on the following results: i) The cellular morphology changed from adherent to scattered; ii) the level of EMT-associated biomarkers were altered (E-cadherin declined and vimentin increased); and iii) the migration potential increased. These results are in accordance with those of previous studies, which demonstrated that EMT markers change at the molecular level in colon cancer cells $(22,23)$. 
Therefore, the present study provided evidence that 5-FU resistance is associated with the EMT.

The EMT is a complex process that involves several signaling pathways. A number of studies have reported an overexpression of EMT-associated signaling pathways, including the Notch, Wnt and nuclear factor- $\kappa \mathrm{B}$ in human colon cancer cells $(24,25)$. In order to determine which pathway(s) are involved in the process of drug resistance in HCT-8/5-FU cells, the present study used inhibitors of the Notch (DAPT), TGF- $\beta$ (LY2109761), PI3K (LY294002), extracellular-signal-regulated kinase (UO126) and Hedgehog (GDC0449) signaling pathways. The results identified that the inhibitor of the Hedgehog signaling pathway, GDC0449, was most effective at reversing drug resistance, with $5 \mu \mathrm{M}$ determined to be the optimal concentration of GDC0449. In addition, EMT biomarkers were analyzed using RT-PCR. The results demonstrated that the EMT-associated biomarker, $\mathrm{N}$-cadherin, was downregulated.

In conclusion, the present study revealed that $5-\mathrm{FU}$ induces an EMT phenotype in HCT-8/5-FU colon cancer cells and that this change is associated with the Hedgehog signaling pathway. The Hedgehog inhibitor, GDC0449, increased drug sensitivity. These findings present a novel clinical therapy for the treatment of colon cancer with acquired resistance to 5 -FU.

\section{Acknowledgements}

This study was supported by grants from the Program for New Century Excellent Talents in University of The Ministry of Education of China (no. NCET-12-0880), the Fundamental Research Funds for the Central Universities (no. JUSRP51311A), the China National Natural Science Foundation grants (nos. 81100185 and 81273437) and an NSFC-RGC joint grant (no. 81361168001).

\section{References}

1. Cunningham D, Atkin W, Lenz HJ, et al: Colorectal cancer. Lancet 375: 1030-1047, 2010.

2. Verdecchia A, Francisci S, Brenner H, et al; EUROCARE-4 Working Group: Recent cancer survival in Europe: a 2000-02 period analysis of EUROCARE-4 data. Lancet Oncol 8: 784-796, 2007.

3. No authors listed: Efficacy of adjuvant fluorouracil and folinic acid in colon cancer. International Multicentre Pooled Analysis of Colon Cancer Trials (IMPACT) investigators. Lancet 345: 939-944, 1995.

4. Rutman RJ, Cantarow A and Paschkis KE: Studies in 2-acetylaminofluorene carcinogenesis. III. The utilization of uracil-2-C14 by preneoplastic rat liver and rat hepatoma. Cancer Res 14: $119-123,1954$.

5. Thomas DM and Zalcberg JR: 5-fluorouracil: a pharmacological paradigm in the use of cytotoxics. Clin Exp Pharmacol Physiol 25: 887-895, 1998.
6. Noordhuis P, Holwerda U, Van der Wilt CL, et al: 5-Fluorouracil incorporation into RNA and DNA in relation to thymidylate synthase inhibition of human colorectal cancers. Ann Oncol 15: 1025-1032, 2004

7. Zhang N, Yin Y, Xu SJ and Chen WS: 5-Fluorouracil: mechanisms of resistance and reversal strategies. Molecules 13: 1551-1569, 2008.

8. Giacchetti S, Perpoint B, Zidani R, et al: Phase III multicenter randomized trial of oxaliplatin added to chronomodulated fluorouracil-leucovorin as first-line treatment of metastatic colorectal cancer. J Clin Oncol 18: 136-147, 2000.

9. Douillard JY, Cunningham D, Roth AD, et al: Irinotecan combined with fluorouracil compared with fluorouracil alone as first-line treatment for metastatic colorectal cancer: a multicentre randomised trial. Lancet 355: 1041-1047, 2000.

10. De Wever O, Pauwels P, De Craene B, et al: Molecular and pathological signatures of epithelial-mesenchymal transitions at the cancer invasion front. Histochem Cell Biol 130: 481-494, 2008.

11. Thiery JP: Epithelial-mesenchymal transitions in tumour progression. Nat Rev Cancer 2: 442-454, 2002.

12. Rosanò L, Cianfrocca R, Spinella F, et al: Acquisition of chemoresistance and EMT phenotype is linked with activation of the endothelin A receptor pathway in ovarian carcinoma cells. Clin Cancer Res 17: 2350-2360, 2011.

13. Kurokawa M, Ise N, Omi K et al: Cisplatin influences acquisition of resistance to molecular-targeted agents through epithelial-mesenchymal transition-like changes. Cancer Sci 104: 904-911, 2013.

14. Chen Y, Sun Y, Chen L, et al: miRNA-200c increases the sensitivity of breast cancer cells to doxorubicin through the suppression of E-cadherin-mediated PTEN/Akt signaling. Mol Med Rep 7: 1579-1584, 2013

15. Huang XY, Ke AW, Shi GM, et al: $\alpha \mathrm{B}$-crystallin complexes with $14-3-3 \xi$ to induce epithelial-mesenchymal transition and resistance to sorafenib in hepatocellular carcinoma. Hepatology 57: 2235-2247, 2013.

16. Zhou Q, Zeng R, Xu C, et al: Erbin inhibits TGF- $\beta 1$-induced EMT in renal tubular epithelial cells through an ERK-dependent pathway. J Mol Med (Berl) 90: 563-574, 2012.

17. Conti B, Minutolo A, Arciello M and Balsano C: Are Hedgehog and Wnt/ $\beta$-catenin pathways involved in hepatitis $C$ virus-mediated EMT? J Hepatol 58: 636-637, 2013.

18. Xu X, Zhou Y, Xie C, et al: Genome-wide screening reveals an EMT molecular network mediated by Sonic hedgehog-Gli1 signaling in pancreatic cancer cells. PLoS One 7: e43119, 2012.

19. Shan D, Chen L, Njardarson JT, et al: Synthetic analogues of migrastatin that inhibit mammary tumor metastasis in mice. Proc Natl Acad Sci USA 102: 3772-3776, 2005.

20. Shan D, Chen L, Wang D, Tan YC, Gu JL and Huang XY: The $\mathrm{G}$ protein $\mathrm{G}$ alpha(13) is required for growth factor-induced cell migration. Dev Cell 10: 707-718, 2006.

21. Yang $\mathrm{S}$ and Huang XY: $\mathrm{Ca} 2^{+}$influx through L-type $\mathrm{Ca} 2^{+}$channels controls the trailing tail contraction in growth factor-induced fibroblast cell migration. J Biol Chem 280: 27130-27137, 2005.

22. Todosi AM, Gavrilescu MM, Aniţei GM, et al: Colon cancer at the molecular level - usefulness of epithelial-mesenchymal transition analysis. Rev Med Chir Soc Med Nat Iasi 116: 1106-1111, 2012.

23. Bhangu A, Wood G, Mirnezami A, Darzi A, Tekkis P and Goldin R: Epithelial mesenchymal transition in colorectal cancer: Seminal role in promoting disease progression and resistance to neoadjuvant therapy. Surg Oncol 21: 316-323, 2012.

24. Jin H, Gong W, Zhang C and Wang S: Epigallocatechin gallate inhibits the proliferation of colorectal cancer cells by regulating Notch signaling. Onco Targets Ther 6: 145-153, 2013.

25. Wang FX, Deng AJ, Li M, Wei JF, Qin HL and Wang AP: (3S)-1, 2,3,4-Tetrahydro- $\beta$-carboline-3-carboxylic acid from Cichorium endivia. L induces apoptosis of human colorectal cancer HCT-8 cells. Molecules 18: 418-429, 2012. 